\title{
Orientational and Crystalline Order of Copper-Phthalocyanine Films on Gold: the Role of Substrate Roughness and Cleanliness
}

\author{
Michael Kothe and Gregor Witte* \\ Molekulare Festkörperphysik, Philipps-Universität Marburg, 35032 Marburg, Germany \\ *E-Mail: gregor.witte@physik.uni-marburg.de
}

\section{Contents:}

1. C1s NEXAFS Signature and Geometrical Structure of CuPc S2

2. Visualization of CuPc Lattice Planes Observed by XRD S3

3. Mosaicity of CuPc Films on Au(111) and Au(poly) S4

4. Influence of Growth Temperature on CuPc Films on Polycrystalline Gold S5

5. Morphology and Side Faces of Individual CuPc Crystallites S6 


\section{C1s NEXAFS Signature and Geometrical Structure of CuPc}

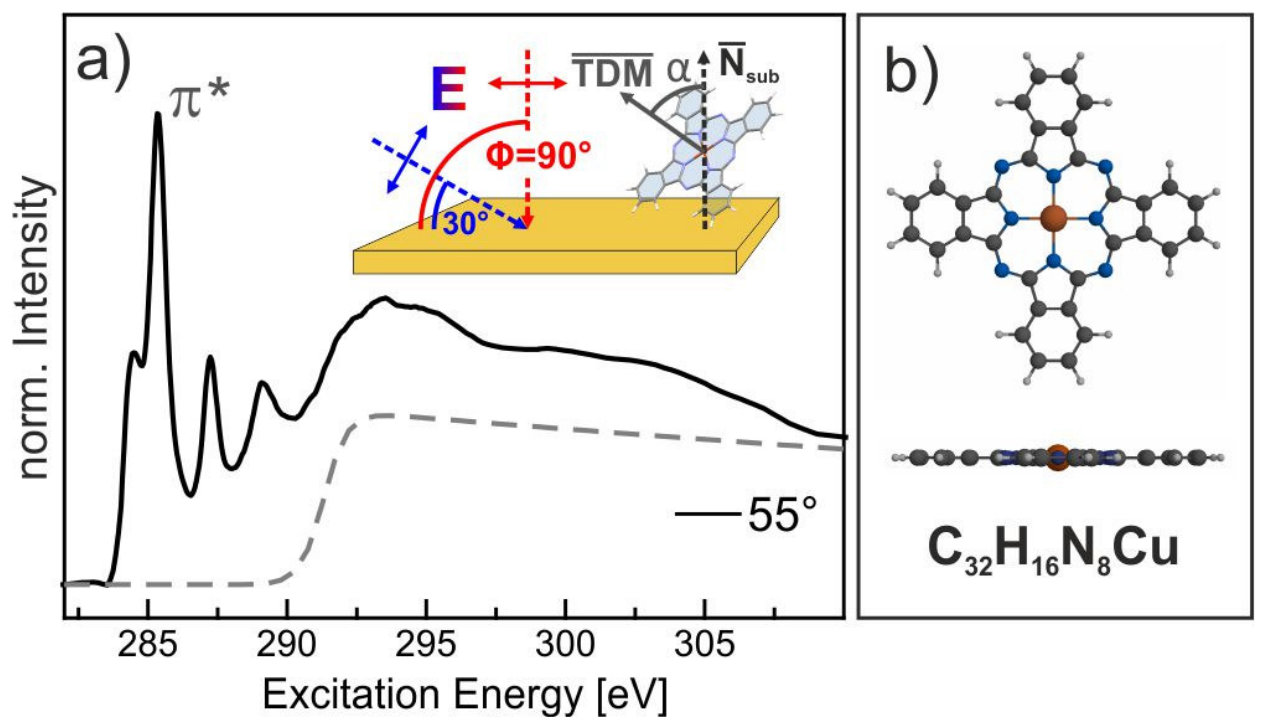

Figure S1: (a) C1s overview NEXAFS signature of a $1.5 \mathrm{~nm}$ CuPc film grown on $\mathrm{Au}(111)$, together with the corresponding experimental setup schematically shown in the inset. The gray-dotted line indicates the position of the C1s ionization edges. (b) Top and side view of the geometrical structure of a Copper-(II)-Phthalocyanine molecule.

Fig. S1a depicts a C1s overview NEXAFS spectrum of a thin CuPc film, that is recorded at the magic angle of incidence $\Phi=55^{\circ}$ where the intensity of the different resonances is largely independent on the angle of incidence. As shown in previous studies the leading resonances below the C1s ionization edges (cf. gray dotted line) are due to transitions from C1s core levels into unoccupied molecular orbitals of $\pi^{*}$-type, while resonances at energies above the absorption edge are mainly due to transitions into unoccupied molecular $\sigma^{*}$-orbitals. ${ }^{\mathrm{S1}}$

The inset of panel (a) depicts the relevant angles of the experimental setup, with the X-ray incidence angle $\Phi$ and the angle between the surface normal and the corresponding transition dipole moment $\alpha$, which is identical to the angle between the molecular plane and substrate surface in the case of vector-type $\pi^{*}$-transitions. Figure $\mathrm{S} 1 \mathrm{~b}$ presents the molecular structure of CuPc. The side view indicates the planar nature of CuPc, which validates the assumption of vector-type $\pi^{*}$-transitions. 


\section{Visualization of CuPc Lattice Planes Observed by XRD}

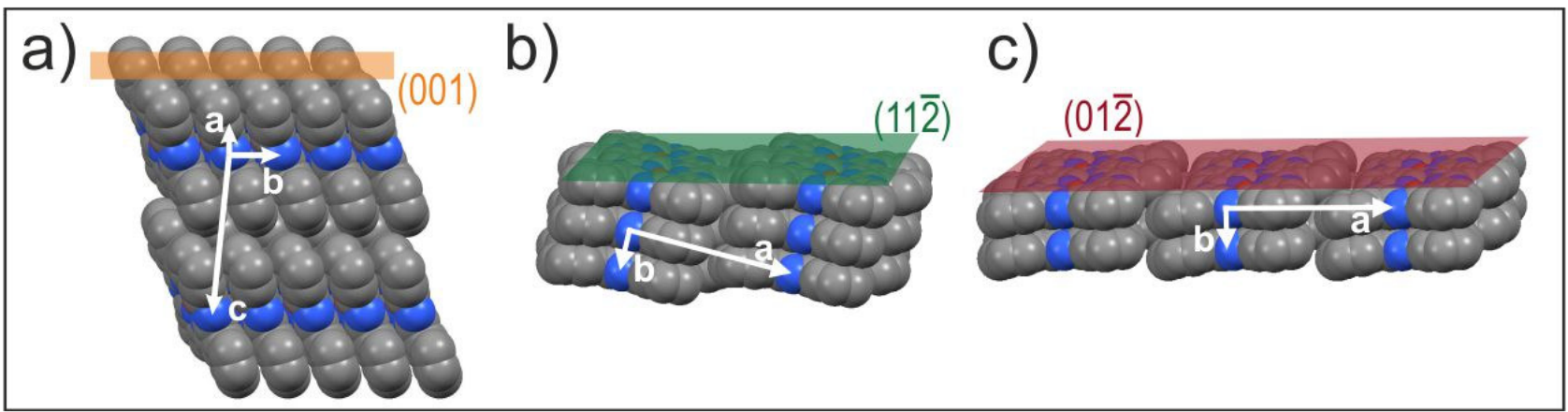

Figure S2: Molecular arrangement of the molecules in $\alpha$-CuPc polymorph ${ }^{1}$ and the corresponding lattice planes observed

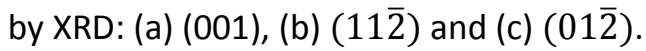

Fig S2 depicts the molecular arrangements of the various CuPc lattice planes that were observed in the out-ofplane X-ray measurements (cf. Figs. 2 and 3), based on the $\alpha$-polymorph crystal structure reported by (Hoshino et al.)..$^{\mathrm{s}}$

In the (001) plane molecules are uprightly oriented, while in $(11 \overline{2})$ and $(01 \overline{2})$ planes molecules adopt a recumbent orientation with a tilt of the molecular plane of about $7^{\circ}$ with respect to the crystallographic planes. Please note that the observed $\alpha$-polymorph (Hoshino et $a l$. $)^{52}$ is different to the $\alpha$-polymorph reported by Ashida et al., ${ }^{\text {S3 }}$ which is also frequently cited in literature. 


\section{Mosaicity of CuPc Films on Au(111) and Au(poly)}

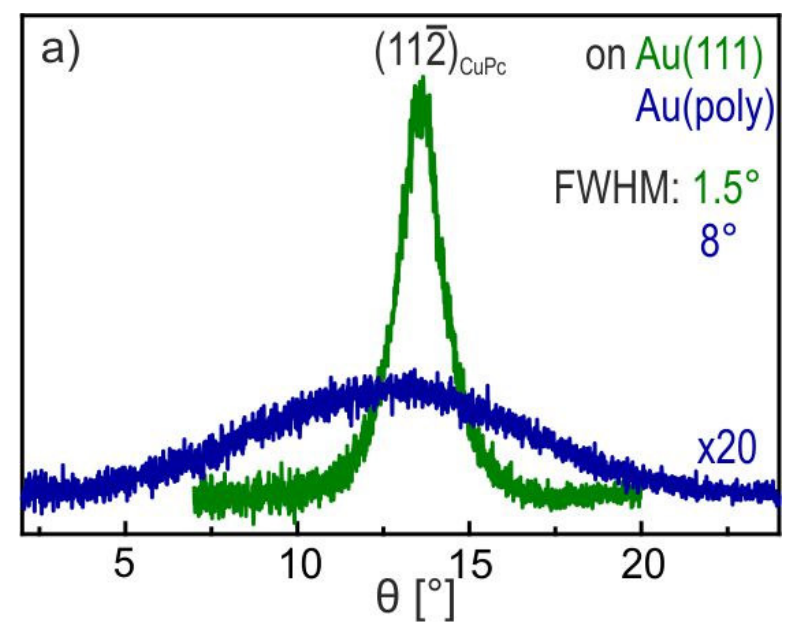

Figure S3: XRD rocking curves of the (11) $)$-reflections, recorded for $50 \mathrm{~nm}$ CuPc films grown at $350 \mathrm{~K}$ on clean Au(111) (green) and polycrystalline gold (blue) surfaces, respectively.

The NEXAFS dichroism analysis of CuPc revealed a larger molecular tilt angle for films grown on polycrystalline gold surface as compared to that on the $\mathrm{Au}(111)$ surface. In order to better understand this effect, also rocking curves of the $(11 \overline{2})$-reflection were recorded for $50 \mathrm{~nm}$ CuPc films grown on the different gold surfaces. This yields a rocking width of $1.5^{\circ}$ for $\mathrm{CuPc}$ on $\mathrm{Au}(111)$ while on polycrystalline gold a significantly larger value of $8^{\circ}$ is found, which reflect larger mosaicity on polycrystalline gold and partly explains the larger effective tilt angle found by the NEXAFS analysis. 


\section{Influence of Growth Temperature on CuPc Films on Polycrystalline Gold}
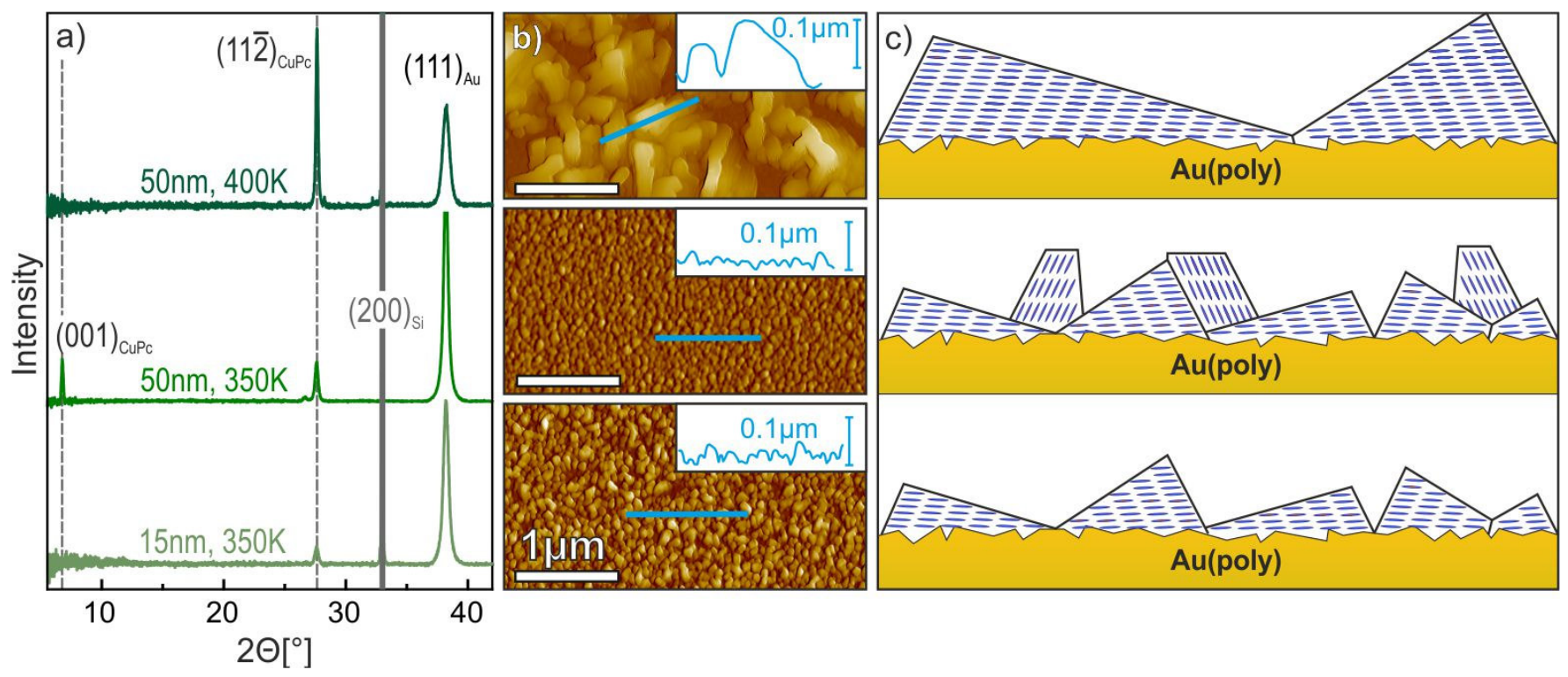

Figure S4: Influence of growth temperature and film thickness on (a) the resulting crystalline texture and (b) morphology of CuPc films grown on clean polycrystalline gold substrates that is schematically summarized in (c).

Figure $2 \mathrm{~d}$ of the main paper shows that a $50 \mathrm{~nm}$ thick CuPc film grown at $350 \mathrm{~K}$ on polycrystalline gold consists of regions of recumbent as well as standing molecules. To find out whether both orientations are adopted right from the beginning or only with increasing film thickness, we have also compared the crystalline structure and morphology of $15 \mathrm{~nm}$ and $50 \mathrm{~nm}$ thick films grown at $350 \mathrm{~K}$. As shown in Figures $\mathrm{S} 4$ the thinner film exclusively reveals the $(11 \overline{2})$-reflection indicating solely a recumbent orientation, while for further increased film thickness also the (001)-reflection appears which evidences an upright molecular orientation. Interestingly, at the same time the rms film roughness is reduced from $12 \mathrm{~nm}$ to $8 \mathrm{~nm}$, which shows that the crystalline domains with upright orientation are distinctly smaller and thereby reduce the film roughness.

By contrast, when increasing the substrate temperature during deposition to $400 \mathrm{~K}$ the formation of CuPc domains with upright molecular orientation on the clean polycrystalline gold substrate is efficiently suppressed and instead leads to the formation of large CuPc crystallites. These (11) $)$-oriented crystallites have an oblique shape with lateral sizes of up to $1 \mu \mathrm{m}$ and a height of more than $100 \mathrm{~nm}$ for a nominal film thickness of $50 \mathrm{~nm}$. While the crystallinity of the CuPc film formed at elevated temperature is increased and molecules homogeneously adopt a recumbent orientation, the formation of individual crystallites largely increases the film roughness yielding an $r m s$ value of $42 \mathrm{~nm}$. 


\section{Morphology and Side Faces of Individual CuPc Crystallites}

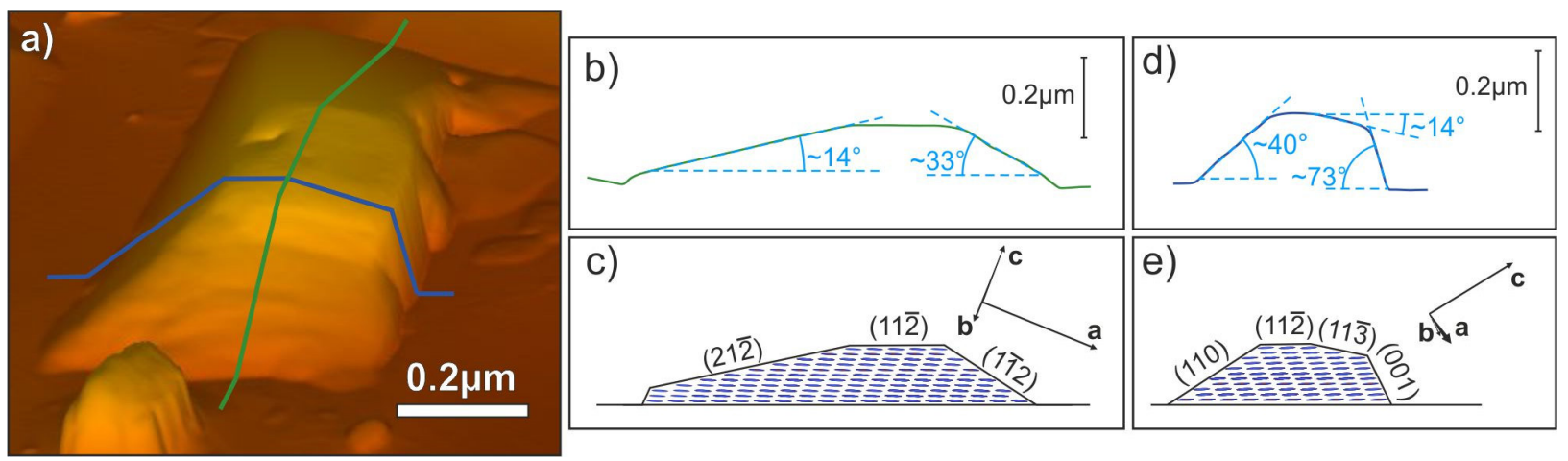

Figure S5: Morphological analysis of isolated CuPc crystallites formed upon film growth at elevated temperatures on $\mathrm{Au}(111)$. (a) 3D-AFM micrograph of a typical crystallite ( $50 \mathrm{~nm}$ grown at $450 \mathrm{~K}, \mathrm{cg}$. Figure 1f), together with (b,d) line scans (green and blue) and an analysis of the slope of the characteristic side faces. These angles can be attributed to specific crystallographic surface planes, that are shown in panels (c,e).
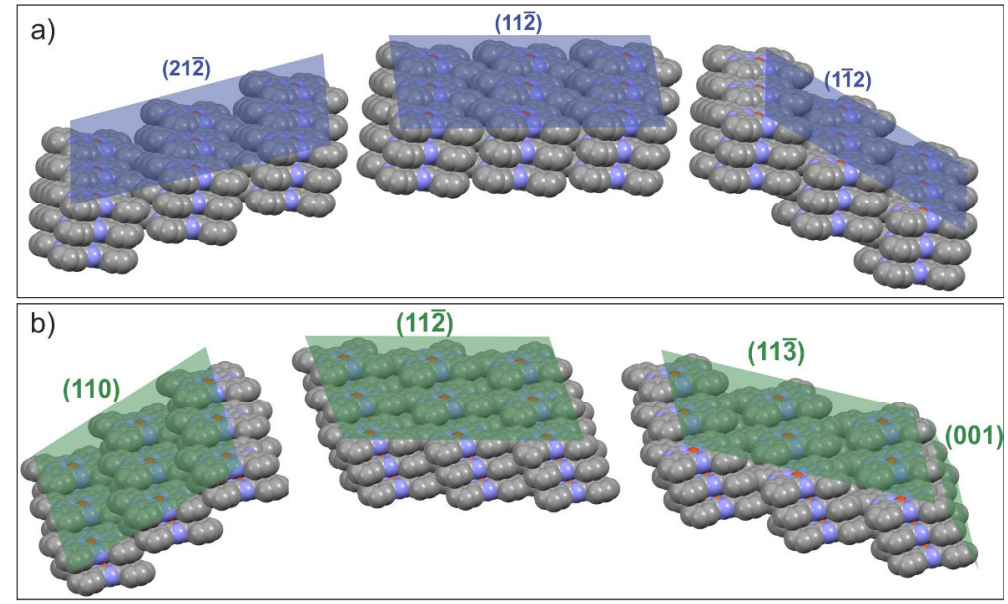

Figure S6: Visualization of molecular packing motifs of the various side faces observed for the CuPc crystallites.

Since the thermodynamic equilibrium shape of crystals is largely determined by the surface free energies of the limiting side faces, ${ }^{, 4, S 5}$ AFM data are used to determine the characteristic side faces of individual crystallites formed upon growth at elevated temperature (cf. Figure $1 \mathrm{f}$ in main paper). As shown in Figure S5a the side faces of CuPc crystallites exhibit characteristic angles with respect to the (11/2) contact plane which can be welldescribed by specific crystallographic lattice planes of the $\alpha$-phase. Figure $\$ 6$ depicts the molecular packing motifs in such planes which shows a rather dense packing and similar coordination which corroborates the idea that they have comparable surface energies which favors the formation of such distinct oblique crystallites and in turn causes a notable film roughness. 


\section{References}

(S1) De Francesco, R.; Stener, M.; Fronzoni, G. Theoretical Study of Near-Edge X-Ray Absorption Fine Structure Spectra of Metal Phthalocyanines at C and N K-Edges. J. Phys. Chem. A 2012, 116, 2885-2894.

(S2) Hoshino, A.; Takenaka, Y.; Miyaji, H. Redetermination of the Crystal Structure of $\alpha$-Copper Phthalocyanine Grown on KCl. Acta Crystallogr., Sect. B: Struct. Sci., Cryst. Eng. Mater. 2003, 59, 393-403.

(S3) Ashida, M.; Uyeda, N.; Suito, E. Unit Cell Metastable-form Constants of Various Phthalocyanines Bull. Chem. Soc. Jpn. 1966, 39, 2616-2624.

(S4) Northrup, J. E.; Tiago, M. L.; Louie, S. G. Surface Energetics and Growth of Pentacene Phys. Rev. B 2002, 66, 121404(R).

(S5) Nabok, D.; Puschnig, P.; Ambrosch-Draxl, C. Cohesive and Surface Energies of $\pi$-Conjugated Organic Molecular Crystals: A First-Principles Study. Phys. Rev. B 2008, 77, 245316. 